\title{
Shame and depression: The roles of self-reassurance and social safeness
}

\author{
Joana Marta-Simões, M.S. \\ Cláudia Ferreira, M.S., Ph.D \\ Ana Laura Mendes, M.S.
}

Cognitive and Behavioural Center for Research and Intervention, University of Coimbra

\begin{abstract}
Self-reassurance and social safeness are both positive factors linked with a lighter experience of shame and depression symptoms. Self-reassurance is defined as an adaptive emotion regulation process, and social safeness as an emotion experience related to feelings of being safe around others, accepted by others, and connected to one's social world. Nevertheless, data about how self-reassurance and social safeness and pleasure operate in the association between external shame and depression is still scarce. A path model which hypothesised that self-reassurance and social safeness and pleasure may act as mediators on the association between shame and depressive symptomatology was tested. This study's sample consisted of adult men $(n=54)$ and women $(n=125)$, from the Portuguese general population. Results indicated that self-reassurance and social safeness act as mediators in the relationship between shame and depression symptoms. Specifically, a higher report of shame seems to explain higher levels of depressive symptomatology, via lower tendency for self-reassurance and poorer experience of social safeness. The tested model explained $45 \%$ of the variance of depressive symptomatology and was revealed to be invariant between men and women. This study's results underline the profound impact of the experience of comfort within secure and warm social
\end{abstract}


relationships, but mostly the importance of self-soothing and self-compassion abilities which associate with greater social functioning. Moreover, in practical terms, these findings reinforce the pertinence of cultivating self-compassion, which has proven to be particularly relevant when intervening with high levels of shame, and in the prevention of depression. 